\title{
The number of directions determined by less than $q$ points
}

\author{
Szabolcs L. Fancsali • Peter Sziklai • \\ Marcella Takáts
}

Received: 9 September 2011 / Accepted: 5 March 2012 / Published online: 30 March 2012

(C) Springer Science+Business Media, LLC 2012

\begin{abstract}
In this article we prove a theorem about the number of directions determined by less then $q$ affine points, similar to the result of Blokhuis et al. (in J. Comb. Theory Ser. A 86(1), 187-196, 1999) on the number of directions determined by $q$ affine points.
\end{abstract}

Keywords Projective plane $\cdot$ Directions $\cdot$ Polynomials

\section{Introduction}

In this article, $p$ is a prime, and $q=p^{h}$, where $h \geq 1 . \operatorname{GF}(q)$ denotes the finite field with $q$ elements, and $\mathbb{F}$ can denote an arbitrary field (or maybe a Euclidean ring). $\mathrm{PG}(d, q)$ denotes the projective geometry of dimension $d$ over the finite field $\operatorname{GF}(q)$. $\mathrm{AG}(d, q)$ denotes the affine geometry of dimension $d$ over $\operatorname{GF}(q)$ that corresponds to the coordinate space $\operatorname{GF}(q)^{d}$ of rank $d$ over $\operatorname{GF}(q)$.

For the affine and projective planes $\operatorname{AG}(2, q) \subset \mathrm{PG}(2, q)$, we imagine the line $\ell_{\infty}=\mathrm{PG}(2, q) \backslash \mathrm{AG}(2, q)$ at infinity as the set $\ell_{\infty} \cong \mathrm{GF}(q) \cup\{\infty\}$. So the nonvertical directions are field elements (numbers), and the vertical direction is $\infty$.

The original problem of direction sets was the following. Let $f: \operatorname{GF}(q) \rightarrow \operatorname{GF}(q)$ be a function, and let $\mathcal{U}=\{(x, f(x)) \mid x \in \mathrm{GF}(q)\} \subseteq \mathrm{AG}(2, q)$ be the graph of the function $f$. The question is how many directions can be determined by the graph of $f$ ?

S.L. Fancsali · P. Sziklai $(\bowtie) \cdot$ M. Takáts

Dept. of Computer Science, Institute of Mathematics, Eotvos Lorand University, Budapest, Pazmany P. s. 1/c, Budapest 1117, Hungary

e-mail: sziklai@cs.elte.hu 
Definition (Direction set) If $\mathcal{U}$ is an arbitrary set of points in the affine plane $\mathrm{AG}(2, q)$, then we say that the set

$$
\mathcal{D}=\left\{\frac{b-d}{a-c} \mid(a, b),(c, d) \in \mathcal{U},(a, b) \neq(c, d)\right\}
$$

is the set of directions determined by $\mathcal{U}$. We define $\frac{a}{0}$ as $\infty$ if $a \neq 0$; thus $\mathcal{D} \subseteq \operatorname{GF}(q) \cup$ $\{\infty\}$. If $\mathcal{U}$ is the graph of a function, then it simply means that $|\mathcal{U}|=q$ and $\infty \notin \mathcal{D}$.

Simeon Ball [1] proved a stronger version of the structure theorem of Aart Blokhuis, Simeon Ball, Andries Brouwer, Leo Storme and Tamás Szőnyi [3]. To recall their result, we need some definitions.

Definition Let $\mathcal{U}$ be a set of points of $\operatorname{AG}(2, q)$. If $y \in \ell_{\infty}$ is an arbitrary direction, then by $s(y)$ we denote the greatest power of $p$ such that each line $\ell$ of direction $y$ meets $\mathcal{U}$ in zero modulo $s(y)$ points. In other words,

$$
s(y)=\operatorname{gcd}\left(\left\{|\ell \cap \mathcal{U}| \mid \ell \cap \ell_{\infty}=\{y\}\right\} \cup\left\{p^{h}\right\}\right) .
$$

Let $s$ be the greatest power of $p$ such that each line $\ell$ of direction in $\mathcal{D}$ meets $\mathcal{U}$ in zero modulo $s$ points. In other words,

$$
s=\underset{y \in \mathcal{D}}{\operatorname{gcd}} s(y)=\min _{y \in \mathcal{D}} s(y) .
$$

Note that $s(y)$ and thus also $s$ may be equal to 1 . Note that $s(y)=1$ for each nondetermined direction $y \notin \mathcal{D}$.

Remark 1 Suppose that $s \geq p$. Then, for each line $\ell \subset \mathrm{PG}(2, q)$ :

$$
\begin{array}{ll}
\text { either } & (\mathcal{U} \cup \mathcal{D}) \cap \ell=\emptyset \\
\text { or } & |(\mathcal{U} \cup \mathcal{D}) \cap \ell| \equiv 1 \quad(\bmod s) .
\end{array}
$$

Moreover, $|\mathcal{U}| \equiv 0(\bmod s)$.

(If $s=1$, then $0 \equiv 1(\bmod s)$, so in this case the remarks above would be meaningless.)

Proof Fix a direction $y \in \mathcal{D}$. Each affine line with slope $y$ meets $\mathcal{U}$ in zero modulo $s$ points, so $|\mathcal{U}| \equiv 0(\bmod s)$.

An affine line $L \subset \mathrm{AG}(2, q)$ with slope $y \in \mathcal{D}$ meets $\mathcal{U}$ in 0 (mod $s)$ points, so the projective line $\ell=L \cup\{y\}$ meets $\mathcal{U} \cup \mathcal{D}$ in $1(\bmod s)$ points.

An affine line $L \subset \mathrm{AG}(2, q)$ with slope $y \notin \mathcal{D}$ meets $\mathcal{U}$ in at most one point, so the projective line $\ell=L \cup\{y\}$ meets $\mathcal{U} \cup \mathcal{D}$ in either zero or one point.

Let $P \in \mathcal{U}$, and let $L \subset \mathrm{AG}(2, q)$ an affine line with slope $y \in \mathcal{D}$ such that $P \in L$. Then the projective line $\ell=L \cup\{y\}$ meets $\mathcal{U}$ in $0(\bmod s)$ points, and thus, $\ell$ meets $\mathcal{D} \cup \mathcal{U} \backslash\{P\}$ also in $0(\bmod s)$ points. Thus, considering all the lines through $P$ (with slope in $\mathcal{D})$, we get $|\mathcal{U} \cup \mathcal{D}| \equiv 1(\bmod s)$. Since $\mathcal{U}$ has $0(\bmod s)$ points, $|\mathcal{D}| \equiv 1$ $(\bmod s)$. So we get that $\mathcal{U} \cup \mathcal{D}$ meets also the ideal line in $1(\bmod s)$ points. 
Remark 2 (Blocking set of Rédei type) If $|\mathcal{U}|=q$, then each of the $q$ affine lines with slope $y \notin \mathcal{D}$ meets $\mathcal{U}$ in exactly one point, so $\mathcal{B}=\mathcal{U} \cup \mathcal{D}$ is a blocking set meeting each projective line in $1(\bmod s)$ points. Moreover, if $\infty \notin \mathcal{D}$, then $\mathcal{U}$ is the graph of a function, and in this case the blocking set $\mathcal{B}$ above is said to be of Rédei type.

Theorem 3 (Blokhuis, Ball, Brouwer, Storme, and Szőnyi [3]; Ball [1], Theorem 1.1) Let $|\mathcal{U}|=q$ and $\infty \notin \mathcal{D}$. Using the notation $s$ defined above, one of the following holds:

$$
\begin{aligned}
& \text { either } s=1 \quad \text { and } \quad \frac{q+3}{2} \leq|\mathcal{D}| \leq q \text {; } \\
& \text { or } \quad \operatorname{GF}(s) \text { is a subfield of } \operatorname{GF}(q) \quad \text { and } \quad \frac{q}{s}+1 \leq|\mathcal{D}| \leq \frac{q-1}{s-1} \text {; } \\
& \text { or } \quad s=q \quad \text { and }|\mathcal{D}|=1 \text {. }
\end{aligned}
$$

Moreover, if $s>2$, then $\mathcal{U}$ is a $\mathrm{GF}(s)$-linear affine set (of rank $\log _{s} q$ ).

Definition (Affine linear set) A GF(s)-linear affine set is the GF(s)-linear span of some vectors in $\mathrm{AG}(n, q) \cong \mathrm{GF}\left(s^{\log _{s} q}\right)^{n} \cong \mathrm{GF}(s)^{n \log _{s} q}$ (or possibly a translate of such a span). The rank of the affine linear set is the rank of this span over GF $(s)$.

What about the directions determined by an affine set $\mathcal{U} \subseteq \mathrm{AG}(2, q)$ of cardinality not $q$ ? Using the pigeon-hole principle, one can easily prove that if $|\mathcal{U}|>q$, then it determines all the $q+1$ directions. So we can restrict our research to affine sets of less than $q$ points.

Examining the case $q=p$ prime, Tamás Szónyi [7] and later (independently) also Aart Blokhuis [2] have proved the following result.

Theorem 4 (Szőnyi [7], Theorem 5.2; Blokhuis [2]) Let $q=p$ prime and suppose that $1<|\mathcal{U}| \leq p$. Also suppose that $\infty \notin \mathcal{D}$. Then

$$
\begin{array}{ll}
\text { either } & \frac{|\mathcal{U}|+3}{2} \leq|\mathcal{D}| \leq p \\
\text { or } \quad \mathcal{U} \text { is collinear and } \quad|\mathcal{D}|=1
\end{array}
$$

Moreover, these bounds are sharp.

In this article we try to generalize this result to the $q=p^{h}$ prime power case by proving an analogue of Theorem 3 for the case $|\mathcal{U}| \leq q$. Before we examine the number of directions determined by less than $q$ affine points in the plane, we ascend from the plane in the next section and examine the connection between linear sets and direction sets in arbitrary dimensions. The further sections will return to the plane.

\section{Linear sets as direction sets}

The affine space $\mathrm{AG}(n, q)$ and its ideal hyperplane $\Pi_{\infty} \cong \mathrm{PG}(n-1, q)$ of directions together constitute a projective space $\mathrm{PG}(n, q)$. We say that the point $P \in \Pi_{\infty}$ is a 
direction determined by the affine set $\mathcal{U} \subset \mathrm{AG}(n, q)$ if there exists at least one line through $P$ that meets $\mathcal{U}$ in at least two points.

Definition (Projective linear set) Suppose that GF $(s)$ is a subfield of $\mathrm{GF}(q)$. A projective $\mathrm{GF}(s)$-linear set $\mathcal{B}$ of rank $d+1$ is a projected image of the canonical subgeometry $\mathrm{PG}(d, s) \subset \mathrm{PG}(d, q)$ from a center disjoint to this subgeometry. The projection can yield multiple points.

Proposition 5 Suppose that $\mathcal{U}$ is an affine $\mathrm{GF}(s)$-linear set of rank $d+1$ in $\mathrm{AG}(n, q)$ such that $\mathrm{AG}(n, q)$ is the smallest dimensional affine subspace that contains $\mathcal{U}$. Let $\mathcal{D}$ denote the set of directions determined by $\mathcal{U}$. The set $\mathcal{U} \cup \mathcal{D}$ is a projective $\operatorname{GF}(s)$ linear set of rank $d+1$ in $\mathrm{PG}(n, q)$, and all the multiple points are in $\mathcal{D}$.

Proof Without loss of generality, we can suppose that $\mathcal{U}$ contains the origin and suppose that $\mathcal{U}$ is the set of $\operatorname{GF}(s)$-linear combinations of the vectors $\mathbf{a}_{0}, \mathbf{a}_{1}, \ldots, \mathbf{a}_{d}$. We can coordinatize $\operatorname{AG}(n, q)$ so that $\mathbf{a}_{d-n+1}, \ldots, \mathbf{a}_{d}$ is the standard basis of $\operatorname{GF}(q)^{n} \cong$ $\mathrm{AG}(n, q)$.

Embed $\mathrm{GF}(q)^{n} \cong \mathrm{AG}(n, q)$ into $\mathrm{GF}(q)^{d+1} \cong \mathrm{AG}(d+1, q)$ so that $\mathbf{z}_{0}, \mathbf{z}_{1}, \ldots$, $\mathbf{z}_{d-n}, \mathbf{a}_{d-n+1}, \ldots, \mathbf{a}_{d}$ is the standard basis. Let $\pi$ denote the projection of $\mathrm{AG}(d+$ $1, q)$ onto $\operatorname{AG}(n, q)$ such that $\pi\left(\mathbf{z}_{i}\right)=\mathbf{a}_{i}$ for each $i=0, \ldots, d-n$ and $\pi\left(\mathbf{a}_{j}\right)=\mathbf{a}_{j}$ for each $j>d-n$. Then $\mathcal{U}$ is the image of the canonical subgeometry $\operatorname{AG}(d+1, s)$ by $\pi$.

Extend $\pi$ to the ideal hyperplane. The extended $\bar{\pi}$ is a collineation, and so the image of a determined direction is a determined direction. Vice versa, let $A$ and $B$ two arbitrary distinct points in $\ell \cap \mathcal{U}$, and let $P$ be the direction determined by $\pi^{-1}(A)$ and $\pi^{-1}(B)$, then the direction of $\ell$ is $\bar{\pi}(P)$.

Corollary 6 If $\mathcal{D}$ is the set of directions determined by an affine $\mathrm{GF}(s)$-linear set, then $\mathcal{D}$ is a projective $\mathrm{GF}(s)$-linear set.

Remark 7 Olga Polverino [4, Proposition 2.2] proved that if $\mathcal{D}$ is a projective $\operatorname{GF}(s)$ linear set, then $|\mathcal{D}| \equiv 1(\bmod s)$.

The proposition above says that the set of directions determined by an affine linear set is a projective linear set. The converse of this proposition is also true; each projective linear set is a direction set:

Theorem 8 Embed $\mathrm{PG}(n, q)$ into $\mathrm{PG}(n+1, q)$ as the ideal hyperplane and let $\mathrm{AG}(n+$ $1, q)=\mathrm{PG}(n+1, q) \backslash \mathrm{PG}(n, q)$ denote the affine part. For each projective $\mathrm{GF}(s)$ linear set $\mathcal{D}$ of rank $d+1$ in $\mathrm{PG}(n, q)$, there exists an affine $\mathrm{GF}(q)$-linear set $\mathcal{U}$ of rank $d+1$ in $\mathrm{AG}(n+1, q)$ such that the set of directions determined by $\mathcal{U}$ is $\mathcal{D}$.

Proof $\mathcal{D} \subset \mathrm{PG}(n, q)$ is the image of the canonical subgeometry $\mathrm{PG}(d, s) \subset \mathrm{PG}(d, q)$ by the projection $\pi: \mathrm{PG}(d, q) \rightarrow \mathrm{PG}(n, q)$, where the center $C$ of $\pi$ is disjoint to this subgeometry. Embed PG $(d, q)$ into $\mathrm{PG}(d+1, q)$ as the ideal hyperplane and extend $\pi$ to $\bar{\pi}: \mathrm{PG}(d+1, q) \rightarrow \mathrm{PG}(n+1, q)$ so that its center remains $C$. That is, the center is in 
the ideal hyperplane. Consider the canonical subgeometry PG $(d+1, s) \subset \mathrm{PG}(d+1, q)$ and its image by $\bar{\pi}$ :

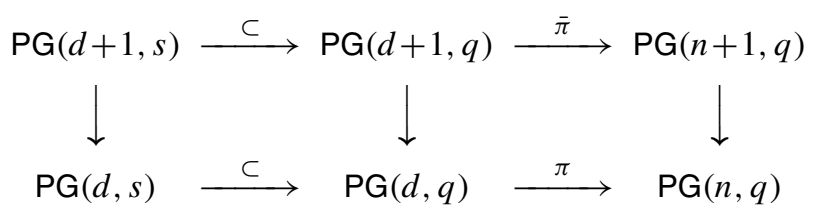

The "ideal part" of this canonical subgeometry $\mathrm{PG}(d, s)$ is the original canonical subgeometry $\mathrm{PG}(d, s)$ of $\mathrm{PG}(d, q)$, and the projection $\bar{\pi}$ project this onto $\mathcal{D}$. Since the center is totally contained in the ideal hyperplane, $\bar{\pi}$ maps the affine part of the canonical subgeometry PG $(d+1, s)$ one-to-one.

The directions determined by the affine part of $\mathrm{PG}(d+1, s)$ are the points of $\mathrm{PG}(d, s)$ in the ideal hyperplane of $\mathrm{AG}(d+1, q)$. Since the extended $\bar{\pi}$ preserves collinearity, the set of directions determined by the projected image of the affine part is $\mathcal{D}$.

\section{The Rédei polynomial of less than $q$ points}

Let $\mathcal{U}$ be a set of less than $q$ affine points in $\operatorname{AG}(2, q)$, and let $\mathcal{D}$ denote the set of directions determined by $\mathcal{U}$. Let $n=|\mathcal{U}|$, and let $R(X, Y)$ be the inhomogeneous affine Rédei polynomial of the affine set $\mathcal{U}$, that is,

$$
R(X, Y)=\prod_{(a, b) \in \mathcal{U}}(X-a Y+b)=X^{n}+\sum_{i=0}^{n-1} \sigma_{n-i}(Y) X^{i},
$$

where the abbreviation $\sigma_{k}(Y)$ means the $k$ th elementary symmetric polynomial of the set $\{b-a Y \mid(a, b) \in \mathcal{U}\}$ of linear polynomials.

Proposition 9 If $y \in \mathcal{D}$, then $R(X, y) \in \mathrm{GF}(q)\left[X^{s(y)}\right] \backslash \mathrm{GF}(q)\left[X^{p \cdot s(y)}\right]$.

If $y \notin \mathcal{D}$, then $R(X, y) \mid X^{q}-X$.

Proof Let $y \in \mathcal{D}$. Then the equation $R(X, y)=0$ has a root $x$ with multiplicity $m$ if there is a line with slope $y$ meeting $\mathcal{U}$ in exactly $m$ points. The value of $x$ determines this line. So each $x$ is either not a root of $R(X, y)$ or a root with multiplicity a multiple of $s(y)$, and $p \cdot s(y)$ does not have this property. Since $R$ is totally reducible, it is the product of its root factors.

If $y \notin \mathcal{D}$, then a line with direction $y$ cannot meet $\mathcal{U}$ in more than one point, so $x$ cannot be a multiple root of $R(X, y)$.

Notation Let $\mathbb{F}$ be the polynomial ring $\mathrm{GF}(q)[Y]$ and consider $R(X, Y)$ as the element of the univariate polynomial ring $\mathbb{F}[X]$. Divide $X^{q}-X$ by $R(X, Y)$ as a univariate polynomial over $\mathbb{F}$; let $Q$ denote the quotient, and let $H+X$ be the negative of the remainder:

$$
Q(X, Y)=\left(X^{q}-X\right) \quad \operatorname{div} R(X, Y) \quad \text { over } \mathbb{F},
$$




$$
-X-H(X, Y) \equiv\left(X^{q}-X\right) \quad \bmod R(X, Y) \quad \text { over } \mathbb{F} .
$$

So

$$
R(X, Y) Q(X, Y)=X^{q}+H(X, Y)=X^{q}+\sum_{i=0}^{q-1} h_{q-i}(Y) X^{i},
$$

where $\operatorname{deg}_{X} H<\operatorname{deg}_{X} R$. Let $\sigma^{*}$ denote the coefficients of $Q$,

$$
Q(X, Y)=X^{q-n}+\sum_{i=0}^{q-n-1} \sigma_{q-n-i}^{*}(Y) X^{i}
$$

and so

$$
h_{j}(Y)=\sum_{i=0}^{j} \sigma_{i}(Y) \sigma_{j-i}^{*}(Y) .
$$

We know that $\operatorname{deg} h_{i} \leq i, \operatorname{deg} \sigma_{i} \leq i$, and $\operatorname{deg} \sigma_{\dot{*}}^{*} \leq i$. Note that the $\sigma^{*}(Y)$ polynomials are not necessarily elementary symmetric polynomials of linear polynomials and if $y \in \mathcal{D}$, then $Q(X, y)$ is not necessarily totally reducible.

Remark 10 Since $\operatorname{deg}_{X} H<\operatorname{deg}_{X} R$, we have $h_{i}=0$ for $1 \leq i \leq q-n$. By definition, $\sigma_{0}=\sigma_{0}^{*}=1$. The equation $h_{1}=0$ implies $\sigma_{1}^{*}=-\sigma_{1}$; this fact and the equation $h_{2}=0$ imply $\sigma_{2}^{*}=-\sigma_{2}+\sigma_{1}^{2}$ and so on, and the $q-n$ equations $h_{i}=0$ uniquely define all the coefficients $\sigma_{i}^{*}$.

Proposition 11 If $y \in \mathcal{D}$, then $Q(X, y), H(X, y) \in \operatorname{GF}(q)\left[X^{s(y)}\right]$, and if $\operatorname{deg} R \leq$ $\operatorname{deg} Q$, then $Q(X, y) \in \mathrm{GF}(q)\left[X^{s(y)}\right] \backslash \mathrm{GF}(q)\left[X^{p \cdot s(y)}\right]$.

If $y \notin \mathcal{D}$, then $R(X, y) Q(X, y)=X^{q}+H(X, y)=X^{q}-X$. In this case $Q(X, y)$ is also a totally reducible polynomial.

Proof If $y \in \mathcal{D}$, then

$$
R(X, y)=X^{n}+\sum_{i=0}^{n-1} \sigma_{n-i}(y) X^{i} \in \mathrm{GF}(q)\left[X^{s(y)}\right] \backslash \mathrm{GF}(q)\left[X^{p \cdot s(y)}\right] .
$$

So $s(y) \mid n$ and $\sigma_{i}(y) \neq 0$ implies $s(y) \mid n-i$ and hence $s(y) \mid i$. The defining equation of $\sigma_{i}^{*}$ contains the sum of products of some $\sigma_{j}$, where the sum of indices (counted with multiplicities) is $i$. Since $\sigma_{j}(y) \neq 0$ only if $s(y) \mid j$, also $\sigma_{i}^{*}(y) \neq 0$ only if $s(y) \mid i$.

If $\operatorname{deg} R \leq \operatorname{deg} Q$, then we can consider $R$ as $\left(X^{q}-X\right) \operatorname{div} Q$, and the reminder is the same $H$.

Since both $R(X, y)$ and $Q(X, y)$ are in $\mathrm{GF}(q)\left[X^{s(y)}\right], H(X, y) \in \mathrm{GF}(q)\left[X^{s(y)}\right]$.

If $y \notin \mathcal{D}$, then $R(X, y) \mid\left(X^{q}-X\right)$ in $\operatorname{GF}(q)[X]$, so $Q(X, y)$ is also totally reducible.

Remark 12 Note that $H(X, y)$ can be an element of $\operatorname{GF}(q)\left[X^{p \cdot s(y)}\right]$. If $H(X, y) \equiv a$ is a constant polynomial, then $R(X, y) Q(X, y)=X^{q}+a=X^{q}+a^{q}=(X+a)^{q}$. 
This means that $R(X, y)=(X+a)^{n}$, and thus, there exists exactly one line (corresponding to $X=-a$ ) of direction $y$ that contains $\mathcal{U}$, and so $\mathcal{D}=\{y\}$.

Definition If $|\mathcal{D}| \geq 2$ (i.e., $H(X, y)$ is not a constant polynomial), then for each $y \in \mathcal{D}$, let $t(y)$ denote the maximal power of $p$ such that $H(X, y)=f_{y}(X)^{t(y)}$ for some $f_{y}(X) \notin \mathrm{GF}(q)\left[X^{p}\right]$ :

$$
H(X, y) \in \mathrm{GF}(q)\left[X^{t(y)}\right] \backslash \mathrm{GF}(q)\left[X^{t(y) p}\right] .
$$

In this case, $t(y)<q$ since $t(y) \leq \operatorname{deg}_{X} H<q$. Let $t$ be the greatest common divisor of the numbers $t(y)$, that is,

$$
t=\underset{y \in \mathcal{D}}{\operatorname{gcd}} t(y)=\min _{y \in \mathcal{D}} t(y) .
$$

If $H(X, y) \equiv a$ (i.e., $\mathcal{D}=\{y\})$, then we define $t=t(y)=q$.

Remark 13 If there exists at least one determined direction $y \in \mathcal{D}$ such that $H(X, y)$ is not constant, then $t<q$. From Proposition 11 we have $s(y) \leq t(y)$ for all $y \in \mathcal{D}$, so $s \leq t$.

Proposition 14 Using the notation above,

$$
R(X, Y) Q(X, Y)=X^{q}+H(X, Y) \in \operatorname{Span}_{\mathbb{F}}\left\langle 1, X, X^{t}, X^{2 t}, X^{3 t}, \ldots, X^{q}\right\rangle .
$$

Proof If $|\mathcal{D}|=\{y\}$, then $H(X, y) \equiv a$ and $H(X, z)=-X$ for $z \neq y$.

Suppose that $|\mathcal{D}| \geq 2$. If $y \notin \mathcal{D}$, then $X^{q}+H(X, y)=X^{q}-X$, and if $y \in \mathcal{D}$, then $X^{q}+H(X, y) \in \mathrm{GF}(q)\left[X^{t(y)}\right] \backslash \mathrm{GF}(q)\left[X^{t(y) p}\right]$.

Thus, in both cases, if $i \neq 1$ and $i \nmid t$, then $h_{q-i}(Y)$ has $q$ roots, and its degree is less than $q$.

\section{Bounds on the number of directions}

Although, in the original problem, the vertical direction $\infty$ was not determined, from now on, without loss of generality, we suppose that $\infty$ is a determined direction (if not, we apply an affine collineation). We continue to suppose that there is at least one nondetermined direction.

Lemma 15 If $\infty \in \mathcal{D} \varsubsetneqq \ell_{\infty}$, then $|\mathcal{D}| \geq \operatorname{deg}_{X} H(X, Y)+1$.

Proof If $y \notin \mathcal{D}$, then $R(X, y) \mid X^{q}-X$; therefore, $H(X, y)=-X$, and thus $\forall i \neq$ $q-1: h_{i}(y)=0$.

If $y \in \mathcal{D}$, then $R(X, y) \nmid X^{q}-X$; hence, $\exists i \neq q-1: h_{i}(y) \neq 0$, and thus $h_{i} \not \equiv 0$. Let $i$ be the smallest index such that $h_{i} \not \equiv 0$, and so $i=q-\operatorname{deg}_{X} H$. Since $h_{i} \not \equiv 0$ has at least $(q+1)-|\mathcal{D}|=q-(|\mathcal{D}|-1)$ roots, $\operatorname{deg}_{Y} h_{i} \geq q-|\mathcal{D}|+1$.

$$
\text { Now } q \geq \operatorname{deg} X^{q-i} h_{i}(Y)=q-i+\operatorname{deg}_{Y} h_{i} \geq 2 q-|\mathcal{D}|+1-i \text {. }
$$


Hence $|\mathcal{D}| \geq q+1-i=\operatorname{deg}_{X} H+1$.

Lemma 16 Let $\kappa(y)$ denote the number of the roots of $X^{q}+H(X, y)$ in $\operatorname{GF}(q)$, counted with multiplicity. If $X^{q}+H(X, y) \neq X^{q}-X$ and if $H(X, y)$ is not a constant polynomial, then

$$
\frac{\kappa(y)-1}{t(y)+1}+1=\frac{\kappa(y)+t(y)}{t(y)+1} \leq t(y) \cdot \operatorname{deg} f_{y}(X)=\operatorname{deg}_{X} H \leq \operatorname{deg} H .
$$

Proof Fix $y \in \mathcal{D}$ and utilize that $X^{q}+H(X, y) \in \operatorname{GF}(q)\left[X^{t(y)}\right]$; thus,

$$
\left(X^{q / t(y)}+f_{y}(X)\right)^{t(y)}=X^{q}+H(X, y)=(a(X) \cdot b(X) \cdot c(X))^{t(y)},
$$

where the totally reducible $a(X)$ contains all the roots (in $\operatorname{GF}(q)$ ) without multiplicity, the totally reducible $b(X)$ contains the further roots (in $\mathrm{GF}(q)$ ), and $c(X)$ has no root in $\operatorname{GF}(q)$. (Note that $t(y)<q$, so $X^{q / t(y)} \in \operatorname{GF}(q)\left[X^{p}\right]$.)

$$
\begin{aligned}
& \left.\begin{array}{l}
a(X) \mid\left(X^{q}-X\right) \\
a(X) \mid\left(X^{q}+f_{y}(X)^{t(y)}\right)
\end{array}\right\} \Longrightarrow a(X) \mid\left(f_{y}(X)^{t(y)}+X\right) \\
& b(X) \mid \partial_{X}\left(X^{q / t(y)}+f_{y}(X)\right)=\partial_{X} f_{y}(X) \\
& a(X) b(X) \mid\left(f_{y}(X)^{t(y)}+X\right) \partial_{X} f_{y}(X) .
\end{aligned}
$$

So, $\operatorname{deg}(a(X) b(X)) \leq t(y) \cdot \operatorname{deg} f_{y}+\operatorname{deg} f_{y}-1=(t(y)+1) \cdot \operatorname{deg} f_{y}-1$, since $\partial_{X} f_{y}(X) \neq 0$ and $f_{y}(X)^{t(y)}=H(X, y) \neq-X$. We get

$$
\frac{\kappa(y)+t(y)}{t(y)+1}=t(y) \frac{\operatorname{deg}(a(X) b(X))+1}{t(y)+1} \leq t(y) \cdot \operatorname{deg} f_{y}(X)
$$

using $\kappa(y)=t(y) \cdot \operatorname{deg}(a(X) b(X))$.

Using these lemmas above, we can prove a theorem similar to Theorem 3 but it is weaker in our case.

Theorem 17 Let $\mathcal{U} \subset \mathrm{AG}(2, q)$ be an arbitrary set of points, and let $\mathcal{D}$ denote the directions determined by $\mathcal{U}$. We use the notation $s$ and $t$ defined above geometrically and algebraically, respectively. Suppose that $\infty \in \mathcal{D}$. One of the following holds:

$$
\begin{aligned}
& \text { either } \quad 1=s \leq t<q \quad \text { and } \quad \frac{|\mathcal{U}|-1}{t+1}+2 \leq|\mathcal{D}| \leq q+1 \text {; } \\
& \text { or } \quad 1<s \leq t<q \quad \text { and } \quad \frac{|\mathcal{U}|-1}{t+1}+2 \leq|\mathcal{D}| \leq \frac{|\mathcal{U}|-1}{s-1} \text {; } \\
& \text { or } \quad 1 \leq s \leq t=q \text { and } \mathcal{D}=\{\infty\} \text {. }
\end{aligned}
$$

Proof The third case is trivial $(t=q$ means $|\mathcal{D}|=1$, by the definition of $t)$.

Let $P$ be a point of $\mathcal{U}$ and consider the lines connecting $P$ and the ideal points of $\mathcal{D}$. Since each such line meets $\mathcal{U}$ and has a direction determined by $\mathcal{U}$, it is incident 
with $\mathcal{U}$ in a multiple of $s$ points. If $s>1$, then counting the points of $\mathcal{U}$ on these lines, we get the upper bound.

If $t<q$, then we can choose a direction $y \in \mathcal{D}$ such that the conditions of Lemma 16 hold. Using Lemma 15 and Lemma 16, we get

$$
|\mathcal{D}| \geq \operatorname{deg}_{X} H(X, Y)+1 \geq \frac{\kappa(y)-1}{t(y)+1}+1+1 .
$$

The number of roots of $R(X, y) Q(X, y)$ is at least the number of roots of $R(X, y)$, which equals $|\mathcal{U}|$. Therefore, $\kappa(y) \geq|\mathcal{U}|$, and thus,

$$
\frac{\kappa(y)-1}{t(y)+1} \geq \frac{|\mathcal{U}|-1}{t+1} \text {. }
$$

An affine collineation converts Szónyi's and Blokhuis' Theorem 4 to the special case of our Theorem 17, since $t$ is equal to either 1 or $p$ in the case $q=p$ prime.

In the case $q>p$, the main problem of Theorem 17 is that the definition of $t$ is nongeometrical. Unfortunately, $t=s$ does not hold in general. For example, let $\mathcal{U}$ be a $\mathrm{GF}(p)$-linear set minus one point. In this case, $s=1$, but $t=p$. In the rest of this article, we try to describe this problem.

\section{Maximal affine sets}

One can easily show that a proper subset of the affine set $\mathcal{U}$ can determine the same directions. (For example, let $\mathcal{U}$ be an affine subplane over the subfield GF $(s)$. Arbitrary $s+1$ points of $\mathcal{U}$ determine the same directions.)

Definition (Maximal affine set) We say that $\mathcal{U} \subseteq \mathrm{AG}(2, q)$ is a maximal affine set that determines the set $\mathcal{D} \subseteq \ell_{\infty} \cong \mathrm{PG}(1, q)$ of directions if each affine set that contains $\mathcal{U}$ as a proper subset determines more than $|\mathcal{D}|$ directions.

Tamás Szőnyi proved a "completing theorem" (stability result) in [6], which was slightly generalized in [5] as follows.

Theorem 18 (Szőnyi [6]; Sziklai [5], Theorem 3.1) Let $\mathcal{D}$ denote the set of directions determined by the affine set $\mathcal{U} \subset \mathrm{AG}(2, q)$ containing $q-\varepsilon$ points, where $\varepsilon<\alpha \sqrt{q}$ and $|\mathcal{D}|<(q+1)(1-\alpha), 1 / 2<\alpha<1$. Then $\mathcal{U}$ can be extended to a set $\mathcal{U}^{\prime}$ with $\left|\mathcal{U}^{\prime}\right|=q$ such that $\mathcal{U}^{\prime}$ determines the same directions.

Szônyi's stability theorem above also stimulates us to restrict ourselves to examine the maximal affine sets only. (An affine set of $q$ points that does not determine all directions is automatically maximal.)

If we examine polynomials in one variable instead of Rédei polynomials, we can get similar "stability" results. Such polynomials occur when we examine $R(X, y)$, $Q(X, y)$, and $H(X, y)$ or $R, Q$, and $H$ over $\operatorname{GF}(q)(Y)$. The second author conjectured that if "almost all" roots of a polynomial $g \in \mathrm{GF}(q)[X]$ have muliplicity a power of $p$, then the quotient $X^{q} \operatorname{div} g$ extends $g$ to a polynomial in $\operatorname{GF}(q)\left[X^{p}\right]$. We can prove more. 
Notation Let $p=$ char $\mathbb{F} \neq 0$ be the characteristic of the arbitrary field $\mathbb{F}$. Let $s=$ $p^{e}$ and $q=p^{h}$ two arbitrary powers of $p$ such that $e \leq h$ (i.e., $s \mid q$, but $q$ is not necessarily a power of $s$ ).

Theorem 19 Let $g, f \in \mathbb{F}[X]$ be polynomials such that $g \cdot f \in \mathbb{F}\left[X^{s}\right]$. If $0 \leq \operatorname{deg} f \leq$ $s-1$, then $X^{q} \operatorname{div} g$ extends $g$ to a polynomial in $\mathbb{F}\left[X^{s}\right]$.

Proof We know that $\operatorname{deg}(g f)=k s(k \in \mathbb{N})$. Let $r=X^{q} \bmod (f g)$ denote the remainder, that is, $X^{q}=(g f) h+r$ where $\operatorname{deg} r \leq \operatorname{deg}(f g)-1$.

Now we show that $h \in \mathbb{F}\left[X^{s}\right]$. Suppose to the contrary that $h \notin \mathbb{F}\left[X^{s}\right]$, i.e., the polynomial $h$ has at least one monomial $\bar{a} \notin \mathbb{F}\left[X^{s}\right]$. Let $\bar{a}$ denote such a monomial of maximal degree. Let $\bar{b}$ denote the leading term of $\mathrm{fg}$. Since $g f \in \mathbb{F}\left[X^{s}\right]$, also $\bar{b} \in \mathbb{F}\left[X^{s}\right]$, and since $\bar{b}$ is the leading term, $\operatorname{deg} \bar{b}=\operatorname{deg}(f g)$. The product $\bar{a} \bar{b}$ is a monomial of the polynomial $(f g) h$, and since $\operatorname{deg} \bar{b}>\operatorname{deg} r, \bar{a} \bar{b}$ is also a monomial of the polynomial $(f \cdot g \cdot h+r)$. The monomial $\bar{a} \bar{b}$ is not in $\mathbb{F}\left[X^{s}\right]$ because $\bar{a} \notin \mathbb{F}\left[X^{s}\right]$ and $\bar{b} \in \mathbb{F}\left[X^{s}\right]$. But $f \cdot g \cdot h+r=X^{q} \in \mathbb{F}\left[X^{s}\right]$, which is a contradiction.

Hence $r \in \mathbb{F}\left[X^{s}\right]$, and so $s \mid \operatorname{deg} r$; thus, if the closed interval $[\operatorname{deg} g, \operatorname{deg}(g f)-1]$ does not contain any integer that is a multiple of $s$, then $\operatorname{deg} r$ is less than $\operatorname{deg} g$.

If we know that $\operatorname{deg} r<\operatorname{deg} g$, then from the equation $X^{q}=(g f) h+r$ we get $X^{q}=g(f h)+r$; hence $r=X^{q} \bmod g$ and $X^{q} \operatorname{div} g=f \cdot h$, where $f$ is a polynomial such that $f g \in \mathbb{F}\left[X^{s}\right]$ and also $h \in \mathbb{F}\left[X^{s}\right]$.

So it is enough to show that the closed interval $[\operatorname{deg} g, \operatorname{deg}(g f)-1]$ does not contain any integer that is a multiple of $s$. Using $\operatorname{deg}(f g)=k s$, we have that the closed interval $[\operatorname{deg} g, \operatorname{deg}(g f)-1]=[k s-\operatorname{deg} f, k s-1]$, and if $0 \leq \operatorname{deg} f \leq s-1$, then it does not contain any integer which is a multiple of $s$.

This theorem above suggests that if the product $R(X, y) Q(X, y)$ is an element of $\mathrm{GF}(q)[Y]\left[X^{p \cdot s(y)}\right]$ while $R(X, y) \in \mathrm{GF}(q)[Y]\left[X^{s(y)}\right] \backslash \mathrm{GF}(q)[Y]\left[X^{p \cdot s(y)}\right]$, then a "completing result" might be in the background. If $\mathcal{U}$ is a maximal affine set, then it cannot be completed, so we conjecture the following.

Conjecture 20 If $\mathcal{U}$ is a maximal affine set that determines the set $\mathcal{D}$ of directions, then $t(y)=s(y)$ for all $y \in \mathcal{D}$ where $t(y)>2$.

Note that there can be maximal affine sets which are not linear.

Example (Nonlinear maximal affine set) Let $\mathcal{U} \subset \mathrm{AG}(2, q)$ be a set, $|\mathcal{U}|=q, s=1$, $q \geq|\mathcal{D}| \geq \frac{q+3}{2}$. In this case, $\mathcal{U}$ cannot be linear because then $s$ would be at least $p$. But $\mathcal{U}$ must be maximal since $q+1$ points in $\mathrm{AG}(2, q)$ would determine all directions. Embed $\mathrm{AG}(2, q)$ into $\mathrm{AG}\left(2, q^{m}\right)$ as a subgeometry. Then $\mathcal{U} \subset \mathrm{AG}\left(2, q^{m}\right)$ is a maximal nonlinear affine set of less than $q^{m}$ points.

However, if $s>2$, we conjecture that the maximal set is linear.

Conjecture 21 If $\mathcal{U}$ is a maximal affine set that determines the set $\mathcal{D}$ of directions and $t=s>2$, then $\mathcal{U}$ is an affine $\mathrm{GF}(s)$-linear set. 
Although we conjecture that the maximal affine sets with $s=t>2$ are linear sets, the converse is not true.

Example (Nonmaximal affine linear set) Let $\mathrm{AG}\left(2, s^{i}\right)$ be a canonical subgeometry of $\mathrm{AG}\left(2, q=s^{i \cdot j}\right)$, and let $\mathcal{U}$ be an affine $\mathrm{GF}(s)$-linear set in the subgeometry AG $\left(2, s^{i}\right)$ that contains more than $s^{i}$ points. Then $\mathcal{U}$ determines the same direction set that is determined by the subgeometry $\mathrm{AG}\left(2, s^{i}\right)$.

Acknowledgements The authors were partially supported by the following grants: OTKA K 81310 and OTKA CNK 77780, ERC, Bolyai and TÁMOP 4.2.1./B-09/KMR-2010-0003.

\section{References}

1. Ball, S.: The number of directions determined by a function over a finite field. J. Comb. Theory, Ser. A 104(2), 341-350 (2003)

2. Blokhuis, A.: Personal communication

3. Blokhuis, A., Ball, S., Brouwer, A.E., Storme, L., Szőnyi, T.: On the number of slopes of the graph of a function defined on a finite field. J. Comb. Theory, Ser. A 86(1), 187-196 (1999)

4. Polverino, O.: Linear sets in finite projective spaces. Discrete Math. 310(22), 3096-3107 (2010)

5. Sziklai, P.: On subsets of GF $\left(q^{2}\right)$ with $d$ th power differences. Discrete Math. 208/209, 547-555 (1999)

6. Szônyi, T.: On the number of directions determined by a set of points in an affine Galois plane. J. Comb. Theory, Ser. A 74(1), 141-146 (1996)

7. Szônyi, T.: Around Rédei’s theorem. Discrete Math. 208/209, 557-575 (1999) 